\title{
Battery Testing with the Calculated Discharge Curve Method-3D Mathematical Model
}

\author{
Aleksandar B. Djordjevic*, Dusan M. Karanovic \\ Mirijevski Bulevar 48, Belgrade, Serbia \\ Email: ${ }^{*}$ aldjordj@open.telekom.rs, ${ }^{*}$ aldjordj@eunet.rs
}

Received 28 December 2014; accepted 10 January 2015; published 15 January 2015

Copyright (C) 2015 by authors and Scientific Research Publishing Inc.

This work is licensed under the Creative Commons Attribution International License (CC BY). http://creativecommons.org/licenses/by/4.0/

c) (i) Open Access

\section{Abstract}

The calculated discharge curve method is based on thermodynamically reversible work: The product of the open-circuit voltage, initial current, and time, i.e., the sum of useful energy and energy losses. A calculated discharge curve is based on the constant step change of the battery voltage in correspondence with a cardinal number set. The essential solution is the transformation of the discharge data voltage vs. time into time vs. voltage using basic equations (three-point operators: power of internal resistance and time), which are valid for all battery electrochemical systems, battery designs and discharge conditions. The mono and multi-cell battery operating conditions consist of the following: 1) The four discharge modes by constant loads: resistor, current, voltage, and power; 2) Two load regimes: Self-driving and device-driving (galvanostat, potentiostat) or battery connection (serial, parallel, combine); and 3) Continual and intermittent discharge. The battery average cell and cell/battery average characteristics, regarding time and capacities, are introduced as the new battery characteristics.

\section{Keywords}

N-Set, Voltage, Time Interval, Time Sub-Intervals, Capacities, Energies

\section{Introduction}

Battery R \& D, manufacture, product acceptance, performance presentation, and exploitation are supported by testing and defining of battery characteristics. Battery testing is performed in accordance with either international (IEC, IEE, DIN, etc.) or internal (laboratory, producer, user, etc.) standards. These standards define battery re-

\footnotetext{
"Corresponding author.
}

How to cite this paper: Djordjevic, A.B. and Karanovic, D.M. (2015) Battery Testing with the Calculated Discharge Curve Method-3D Mathematical Model. Journal of Power and Energy Engineering, 3, 37-52. 
quirements, functional characteristics, test methods and procedures. The Calculated Discharge Curve Method (CDCM) was developed to assist battery engineers in battery characterization [1] [2] at any stage of battery life. The Calculated Discharge Curve Algorithm (CDCA) and 3D mathematical modelling are mathematical tools. In accordance with battery design and parallel and/or series connection, batteries are either mono or multi-cell blocks. CDCM defines the average battery cell as the representative cell of a multi-cell battery that can be compared to other individual cells. A battery electrochemical system requires defining an operating voltage interval, i.e., open circuit, $U_{o} / V$, starting, $V_{o} / V$ and final $V_{n} / V$ voltage. The starting voltage is an instantaneous voltage drop and is excluded from this article. CDCM is used to analyse battery performance: Profiles and capacities of voltage and current, energy on load and energy losses, cycle life, battery comparison with electrochemical systems, design, and production, etc.

The CDCM approach to battery testing is based on the overall energy volume (OEV) as the sum of the energy on load and energy losses. The OEV is in the form of a right parallelepiped. The base of the parallelepiped is the product of the initial voltage and current, UoIo/VA, referred to as the initial power plane (IPP). The parts of the IPP need to be defined with a cell/battery (C/B) electrochemical model. In this article, the parts of the IPP are different types of power: (a) On load; (b) Internal resistance; (c) External resistance; and (d) CDCM, polarisation resistance. During battery discharging, power on load is decreasing, while power losses are increasing. By multiplying the voltage-current, i.e., power surface with time altitude, the energy parallelepiped is defined. CDCA defines time altitudes as follows: (a) Discharge time interval; and (b) Discharge time sub-intervals, the sum of which is the time interval. The cardinal number set $0<i \leq n=N / 2$, named $n$ - $N$-set, defines the constant step change of voltage and corresponds, one to one, to the n-set of time sub-intervals.

CDCA treats discharge time as a dependent variable, i.e., the duration of a predetermined change of battery state of charge. The discharge time interval, $t / s$, is the discharge duration of the open circuit voltage until the actual discharge voltage. A time sub-interval, $\Delta t / s$, is the duration of the voltage change for the constant voltage step. In accordance with the " $\mathrm{S}$ " shape of any discharge curve, the longest time sub-interval lies between the initial and final time sub-interval, $\Delta t_{\text {initial }}<\cdots<\Delta t_{\max }>\cdots>\Delta t_{\text {final }}$. This approach leads to the CDCA basic equation, i.e., time dependence on voltage. Both the discharge time and time sub-intervals, define the OEV parallelepiped in the three forms: (a) The initial power plane multiplied by the discharge time, i.e., thermodynamically reversible energy; (b) The sum of the four right parallelepipeds with power bases (on load and internal, external and CDCM losses) that are multiplied by the discharge time; and (c) The sum of the horizontal layers that have altitudes that are the time sub-intervals and comprise the above four bases, which are changed step by step of discharge, building up the 'hill' of energy on load. Only the sum of the horizontal layers represents the real battery energy balance represented by irregular instead of right parallelepipeds. The known timing of the IPP segments, i.e., discharge time and sub-interval, define the (a) voltage and current capacity, (b) energy on load, and energy losses, which include (c) CDCM, (d) internal and (e) external.

When averaging the battery parameters in regard to time, CDCA introduces the following: average voltage, current, and power, by which an irregular form of the OEV surfaces and spaces are transformed into the regular forms, i.e., parallelograms and parallelepipeds [1] [2]. The battery operating condition comprises 1) four modes of constant load: resistor, current, voltage, and power; 2) two load regimes: self-driving and device-driving (galvanostat, potentiostat or battery connection: serial, parallel, and combined); and 3) continual and intermittent discharge. The five batteries with identical, as much as possible, state of charge and state of health display the five real discharges, self-driving (resistor, current, and voltage) and device-driving (current and voltage) (Table 1$)$.

Table 1. CDCM real and virtual battery discharges.

\begin{tabular}{cccccc}
\hline Parameter & Resistor & Current & Voltage & Power & Battery Response \\
\hline Resistor & Real & Virtual & Virtual & Virtual & Voltage/Current vs. Time \\
Current & Virtual & Real & Virtual & Virtual & Voltage vs. Time \\
Voltage & Virtual & Virtual & Real & Virtual & Current vs. Time \\
Power & Virtual & Virtual & Virtual & Real & Time \\
\hline
\end{tabular}

*Self-driving only; ${ }^{* *}$ Reversible discharge. 
CDCA generates three virtual discharges based on the parameters of the real discharge. The imagined constant power discharge, as the self-driving discharge, is accepted as the reversible discharge. Any cell in serial or parallel connection discharges with serial current or parallel voltage and may display different characteristics in comparison with the discharge across the resistor, i.e., there is a difference between "self" and "device" driving. Any discharge mode may be either continual or intermittent. After the end of continual discharge as well at the end of discharge periods, relaxation curves may be recorded for the new open circuit voltage, i.e., state of charge [1]. Battery discharge parameters, including voltage, current, capacities, energy on load and losses, heat generation, AC impedance, DC resistance, and conductivity, may be monitored during discharge in accordance with the battery test method and data acquisition technique, in addition to being analyzed by CDCM.

\section{CDCM 3D Mathematical Model}

A 3D mathematical model can be used to represent battery electrochemical parts with the CDCA parameters (curves, surfaces and volumes) expressed by algebraic calculations, placing all of them inside the OEV parallelepiped [1] [2]. CDCA is able to determine the OEV with relevant C/B exploitation characteristics of the same electrochemical system and various $\mathrm{C} / \mathrm{B}$ designs, sizes, discharge modes, intensities, etc. Instead of voltagecurrent-time, the $N$-n-time space (where $N \Delta V=U_{o}$ ) may be applied to show parameters of the various electrochemical systems.

\subsection{Power Plane}

CDCA divides the battery discharge voltage interval with constant voltage steps, $\Delta V=U_{o} / N$, to define the voltage sequence:

$$
U_{o}>V_{1}>V_{2}>\cdots>V_{i-1}>V_{i}>V_{i+1}>\cdots>V_{n-2}>V_{n-1}>V_{n}
$$

which is in one-to-one correspondence with the $N$-n-set:

$$
0<1<2<\cdots<(i-1)<i<(i+1)<\cdots<(n-2)<(n-1)<n=\frac{N}{2}
$$

as well as with the over voltage sequence, $\eta_{i}=U_{o}-V_{i}$ :

$$
0<\eta_{1}<\eta_{2}<\cdots<\eta_{i-1}<\eta_{i}<\eta_{i+1}<\cdots<\eta_{n-2}<\eta_{n-1}<\eta_{n}
$$

The sequences of current, $I_{o}>I_{i}>I_{n}$, and current losses, $0<v_{i}<v_{n}$, are calculated with the known load resistor, $\mathrm{Rl} / \mathrm{Ohm}$. In this article, the four electrochemical power segments are as follows:

$$
\begin{aligned}
U_{o} I_{0} & =\left(V_{i}+\eta_{i}\right)\left(I_{i}+v_{i}\right)=V_{i} I_{i}+V_{i} v_{i}+\eta_{i} I_{i}+\eta_{i} v_{i} \\
& =V_{i} I_{i}+V_{i}\left(I_{o}-I_{i}\right)+\left(U_{o}-V_{i}\right) I_{i}+\eta_{i} v_{i}=P_{\text {load }, i}+P_{\text {ext }, i}+P_{\text {int }, i}+P_{\text {CDCM }, i} .
\end{aligned}
$$

on load external internal CDCM and are shown in Figure 1. The next equations represent the step changes of CDCA powers:

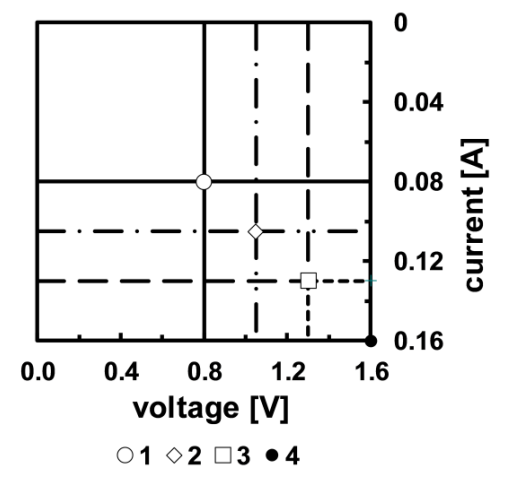

Figure 1. CDCM battery power plane, LR20VARTA, $10 \mathrm{Ohm}, 1$. Final power on load, (VI) n/VA; 2. Actual power on load, (VI)i/VA; 3. Actual average power on load, (VI) avg, i/VA; 4. Monocell battery initial power, UoIo/VA. 


$$
\begin{gathered}
\Delta(V v)_{i}=V_{i} \Delta v+\Delta \eta I_{i}+\Delta \eta \Delta v=2 N \Delta \eta \Delta v+2 i \Delta \eta \Delta v+\Delta \eta \Delta v \\
\Delta\left(V v_{i}\right)_{i}=V_{i} v_{i}-\left(V_{i}+\Delta \eta\right)\left(v_{i}-\Delta v\right)=V_{i} \Delta v-\Delta \eta v_{i}+\Delta \eta \Delta v=(N-2 i+1) \Delta \eta \Delta v \\
\Delta(\eta I)_{i}=\eta_{i} I_{i}-\left(\eta_{i}-\Delta \eta\right)\left(I_{i}+\Delta v\right)=\Delta \eta I_{i}-\eta_{i} \Delta v+\Delta \eta \Delta v=(N-2 i+1) \Delta \eta \Delta v \\
\Delta(\eta v)_{i}=\eta_{i} v_{i}-\left(\eta_{i}-\Delta \eta\right)\left(v_{i}-\Delta v\right)=\eta_{i} \Delta v+\Delta \eta v_{i}-\Delta \eta \Delta v=(2 i-1) \Delta \eta \Delta v
\end{gathered}
$$

$\square$ Overvoltage, $\eta / V$ may be the sum of the components defined by the electrochemical model.

\subsection{OEV Geometric Forms and Parameters}

CDCM accepts the electrochemical definition of discharge curve ([3], p. 59):

"Discharge curves show either the open circuit voltage of a cell or half-cell as a function of the fraction of discharge completed, or, much more commonly, the cell voltage during a deep discharge, usually under a fixed load or at constant current. The abscissa may be calibrated in terms of the quantity of electricity passed (Ah) or as a percentage of the theoretical capacity."

A CDCA discharge curve of any electrochemical system, battery design, discharge mode and regime, and load intensity lies inside an OEV parallelepiped whose base is $U_{o} I_{o}=N^{2} \Delta \eta \Delta v$ CDCA describes the OEV geometrically in accordance with an electrochemical model. The parallelogram " $\Delta \eta \Delta v$ " is the smallest parallelogram of IPP. In this article, $N^{2} \Delta \eta \Delta v$ parallelograms are shared with the four electrochemical parallelograms. The distribution of $N^{2}$ parallelograms between partial parallelograms changes by time, step by step, as the discharge time expires. In the case of constant resistive load, Rl/Ohm, the first discharge time sub-interval, $\Delta t_{1}$, defines the initial horizontal layer: $U_{o} I_{o} \Delta t_{1}$, i.e., $N^{2} \Delta V \Delta I$ base of $\Delta t_{1}$ altitude, which is energy on load only because of the nonexistence of energy losses. During the $\Delta t_{1}$ sub-interval, the power on load is reduced to the $V_{1} I_{1}$ parallelogram, i.e., $(N-1)^{2} \Delta V \Delta I$ parallelograms, and the $(2 N-1)$ parallelograms appear as power losses of the $\Delta t_{2}$ sub-interval, i.e., the second horizontal layer that comprises energies on load and losses. The next layers are in accordance with Equations (5)-(8), and they form the horizontal layers of the decreasing power on load and increasing power of energy losses all the way to the last power layer. The "hill" of the energy on load (see Figure 9) is rising to the $n$-th horizontal layer, $V_{n} I_{n}$. Both hill slides, in an "S" shape, start at an initial voltage and current and are placed until the final layer, which covers one-fourth of the initial power plane. If the " $n$ " successive time altitudes are placed at the line parallel either with the voltage or current axes, they constitute a 2D discharge curve. If the " $n$ " successive time altitudes are placed at any of the power $(V-I, \eta-v)$ lines, they constitute a 3D discharge curve (see Figure 9). The possible battery model, defined by discharge modes and regimes, divides the OEV into the different parts, but all are placed inside the same OEV's volume, as the IPP, discharge time and time sub-intervals remain constant. CDCM battery analysis is based on reversible and irreversible and real and virtual discharges of various electrochemical systems, discharge modes, regimes, and intensities.

\subsubsection{Reversible and Irreversible Discharges}

If the resistive load tends to infinity, thermodynamically reversible battery discharge occurs. An energy balance of irreversible battery discharge emanates from the reversible discharge. A battery and its resistive load may be placed in an adiabatic calorimeter [4]. During the discharge, heat will evolve on the load resistor and the battery. Their sum forms the calorimeter reading, and CDCM considers this energy as battery reversible work. This work is presented as an OEV parallelepiped, inside which CDCA places the energy on load and energy losses. If the battery and resistive load are placed in the two separate calorimeters, the work on the load resistor and the battery heat will be measured, separately, and their sum is equal to the reversible work. CDCA defines energy losses in accordance with the battery model OEV comprises the $n=N / 2$ horizontal energy layers, inside which are the parts of energy on load and energy losses. The OEV parallelepiped base, i.e., the IPP remains constant, whereas the bases of the particular energies, i.e., horizontal layers as well as sub-interval's altitudes, are changed.

\subsubsection{Electrochemical and CDCA Discharge Curve}

Electrochemically, a discharge time curve is expressed by pairs: voltage vs. time. The registered pairs are as 
follows

$$
\begin{gathered}
0<t_{1}<t_{2}<\cdots<t_{i-1}<t_{i}<t_{i+1}<\cdots<t_{n-2}<t_{n-1}<t_{n}=t_{\text {final }} \\
U_{o}>V_{t 1}>V_{t 2}>\cdots>V_{t(i-1)}>V_{t i}>V_{t(i+1)}>\cdots>V_{t(n-2)}>V_{t(n-1)}>V_{t n}=V_{\text {final }}
\end{gathered}
$$

The time is the independent variable, and voltage is the dependent variable. The CDCM approach is reversed: voltage is the independent variable, whereas discharge time is the dependent variable. The CDCA task is to transform voltage vs. time to time vs. voltage. The discharge time interval, $t / s$, is equal to the sum of the time subintervals, $\Delta t / s$. In this manner, an ordered triple is formed:

$$
\begin{gathered}
0<t_{1}<t_{2}<\cdots<t_{i-1} t_{i}<t_{i+1}<\cdots<t_{n-2}<t_{n-1}<t_{n}=t_{\text {final }} \\
0<\Delta t_{1}<\Delta t_{2}<\cdots<\Delta t_{\max -1}<\Delta t_{\max }>\Delta t_{\max +1}>\cdots>\Delta t_{n-1}>\Delta t_{n}>0 \\
0<y_{1}<y_{2}<\cdots<y_{\max -1}<y_{\max }>y_{\max +1}>\cdots>y_{n-1}>y_{n}>y_{\text {final }}
\end{gathered}
$$

where " $y$ " may be a battery parameters. By using these sets, including IPP parameters, the electrochemical and CDCA battery characteristics can be calculated [1] [2] [5]. The CDCA parameters are in one to one correspondence with the $N$-n-set. Time altitudes may be placed at the IPP point, line or power surface in accordance with the battery electrochemical parameters.

\subsubsection{CDCA Input Data}

The input data for battery testing, voltage vs time, may be obtained either as a low-frequency recording/reading (manually registered or graph reading) or as a high-frequency recording/reading. The sets of voltage vs time pairs are as follows:

1. Automatically, $\Delta t_{\text {programmed }}=f(t, V, I, \cdots)$

$$
\begin{gathered}
0<t_{1}<t_{2}<\cdots<t_{j-2}<t_{j-1}<t_{j}<\cdots<t_{k-2}<t_{k-1}<t_{k}=t_{\text {final }}=t_{\text {programmed }} \\
0<\eta_{1}<\eta_{2}<\ldots<\eta_{j-1}<\eta_{j}<\eta_{j+1}<\ldots<\eta_{k-2}<\eta_{k-1}<\eta_{k}=\eta_{\text {final }}
\end{gathered}
$$

2. Published (graphically presented or tabulated), either $\Delta t_{\text {graph }}=$ constant or $\Delta V=$ constant

$$
\begin{gathered}
0<t_{1}<t_{2}<\cdots<t_{j-2}<t_{j-1}<t_{j}<t_{j+1}<t_{j+2}<\cdots<t_{\text {final }-1}<t_{\text {final }}=\sum_{1}^{n} \Delta t_{\text {graph }} \\
0<\eta_{1}<\eta_{2}<\cdots<\eta_{j-1}<\eta_{j}<\eta_{j+1}<\cdots<\eta_{\text {final }-1}<\cdots<\eta_{\text {final }}
\end{gathered}
$$

3. Manually reading: $\Delta t_{\text {beginning }} \Leftrightarrow \Delta t_{\text {between }} \Leftrightarrow \Delta t_{\text {final }}$

$$
\begin{gathered}
0<t_{1}<t_{2}<\cdots<t_{j-1}<t_{j}<t_{j+1}<\cdots<t_{\text {final }-1}<t_{\text {final }} \\
0<\eta_{1}<\eta_{2}<\cdots<\eta_{j-1}<\eta_{j}<\eta_{j+1}<\cdots<\eta_{\text {final }-1}<\eta_{\text {final }}
\end{gathered}
$$

Instead of discharge time, all of the time-dependent characteristics may be CDCA input data.

\subsubsection{Input Data in This Article}

The data of the 16 discharges are the input data in this article:

a CDCA discharge curve from published data [1] on the mono-cell battery LR20VARTA, continual discharge, load 10 Ohm, HP 3054 acquisition unit;

b CDCA discharge data from a published graph [2] concerning Mallory alkaline manganese batteries (T. R. Crompton, Small Batteries, (1982) 52), 10 batteries, battery load, Rl/Ohm, with a battery average cell voltage $(1.6 \mathrm{~V})$ as follows;

1. 9K62, two cells, $U_{o}=3.2 \mathrm{~V}, V_{\text {final }}=1.6 \mathrm{~V}$, battery load/Ohm: 50, 100, 250;

2. PX24, two cells, $U_{o}=3.2 \mathrm{~V}, V_{\text {final }}=1.6 \mathrm{~V}$, battery load/Ohm: 100,166 ;

3. PX21, three cells, $U_{o}=4.8 \mathrm{~V}, V_{\text {final }}=2.4 \mathrm{~V}$, battery load/Ohm: 150, 250;

4. 7K67, four cells, $U_{o}=6.4 \mathrm{~V}, V_{\text {final }}=3.2 \mathrm{~V}$, battery load/Ohm: 100, 250, 500 . 
c Lead-acid battery, A512/24.0 Type No. 889561510, published by Sonnenschein, the five constant current loads discharge data were picked up by published graph. The seven pairs: voltage vs time mark out the "S" shape of the discharge curve (Table 2).

The five discharge time sets, $n=320$, for the constant current loads were generated by the same CDCA procedure as for the primary battery [1] and are shown in Figure 2.

One pair, voltage vs time, needs to be chosen from used data as the first and particular pair for CDCA time generation and needs to satisfy the next criteria:

$$
\eta_{t}=U o-V_{t}(a), \quad i=\eta_{t} / \Delta \eta(b), \quad \Delta \eta=U_{0} / N \quad(c), \quad 0<\eta_{i}<\eta_{n} \quad(d)
$$

The experimental overvoltage value: $\eta_{t} / V$, needs to be placed as close as possible to the predetermined CDCA set value, $\eta_{0}<\eta_{i}<\eta_{n}$. The complete CDCA input data are the following ordered triple:

$$
\begin{gathered}
0<1<2<\cdots<j-2<j-1<j<j+1<j+2<\cdots<j_{n}=N / 2 \\
0<\eta_{1}<\eta_{2}<\cdots<\eta_{j-1}<\eta_{j}<\eta_{j+1}<\cdots<\eta_{n-1}<\eta_{n}=U_{0}-V_{n} \\
0<t_{1}<t_{2}<\cdots<t_{j-1}<t_{j}<t_{j+1}<\cdots<t_{n-2}<t_{n-1}<t_{n}=t_{\text {final }}
\end{gathered}
$$

The " $S$ " profile is marked with the input set of the discharge curve, whereas the generated set is the final and complete discharge curve. Any battery electrochemical parameter may be used instead of the overvoltage in Equation (17b). In this article, the 20 generated discharge curves represent the following: the two electrochemical systems, five primary and one lead-acid battery designs, and constant resistive and current loads. The aim of using such input data is as follows: To (a) verify the CDCA basic equation with four CDCA operators; (b) submit a report about the unique methodology for battery characterization; and (c) establish the parameters vs the common abscissa: voltage and/or $N$-set.

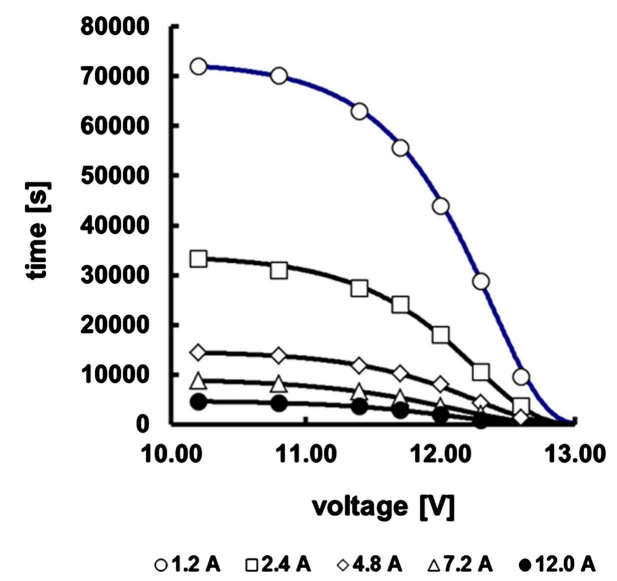

Figure 2. Discharge times vs battery voltage, SONNEN-SCHEIN, lead-acid battery, dryfitA512/24.0 Symbols: input time data (7 points), t/s, Lines: generated time data, t/s, Constant current/A and final times/s: 1.2, 2.4, 4.8, 7.2, 12.0, 72,000, 33,320, 14,400, 8780, 4600.

Table 2. Lead-acid battery discharge data.

\begin{tabular}{cccccc}
\hline LOAD/A & 1.20 & 2.40 & 4.80 & 7.20 & 12.00 \\
\hline $12.6 \mathrm{~V}$ & 9496 & 3600 & 1220 & - & - \\
$12.3 \mathrm{~V}$ & 2889 & 10,437 & 4329 & 2000 & 886 \\
$12.0 \mathrm{~V}$ & 43,846 & 18,056 & 7977 & 3600 & 1853 \\
$11.7 \mathrm{~V}$ & 55,612 & 24,009 & 10,117 & 5300 & 2925 \\
$11.4 \mathrm{~V}$ & 63,000 & 27,341 & 11,820 & 6600 & 3612 \\
$10.8 \mathrm{~V}$ & 70,105 & 30,980 & 13,740 & 8188 & 4300 \\
$10.2 \mathrm{~V}$ & 72,000 & 33,320 & 14,400 & 8780 & 4600 \\
$10.2 \mathrm{~V}$ & 72,000 & 33,320 & 14,400 & 8780 & 4600 \\
\hline
\end{tabular}




\section{CDCA Discharge Time Generation}

\subsection{CDCA Basic Equation}

Investigation of the possible algebraic relation time $=\mathrm{f}$ (voltage, current) is founded on the fact that the IPP is defined by voltage and current intervals as electrochemical system characteristics, as well as on the conclusion that constant voltage steps divide the discharge curve of the "S-shape" into the time intervals, which start as very short and then pass through the maximum values with decreases toward the end. This is in accordance with the citation ([6], p. 181):

"The fundamental curve-fitting problem in two dimensions is to predict from a discrete set of $(x, y)$ data pairs $\left(x_{1}, y_{1}\right)$ through $\left(x_{n}, y_{n}\right)$ the value of $y$ when the value of $x$ is specified. The least-square approximation of Chapter 4 is one form of curve fitting in which we seek the parameters of a given model that best fit the data. In this chapter, we look at other methods, which do not account for random errors in the $x$ and $y$ values. We instead, treat the data as if they were accurate and determine a curve that passes through the data points exactly."

CDCA uses an empirical equation to transform "voltage vs time" into "time vs voltage" to represent CDCM battery testing [1]. In this article, both the (a) experimental 12 pairs' voltage vs. time and (b) the CDCA generated set, $n=320$, LR20VARTA, $10 \mathrm{Ohm}$ time vs voltage [1], are the input data used to derive the CDCA basic equation. The input data, i.e., 12 pairs, have a role in defining the " $S$ " shape of the discharge time, which needs to be satisfied by the CDCA generated time. Between the 12 input pairs, only one pair, voltage vs. time, needs to be chosen as the initial pair for the generation procedure. This chosen pair needs to be placed in correspondence with the CDCA voltage as well as with the $n$-set in the role of the sole common pair of the input and generated set. All other input values have a control role. The generated time curve needs to cover the experimental markers, satisfying the " $S$ " shape. The discharge time generation with the CDCA basic equation, starting from the chosen pair, is performed toward the end as well as at the very beginning of discharge. The three-point time values CDCA operators' member is placed at the " $i$ " position as well as the third, the greater time value.

CDCA derivation of the basic equation is started by defining the power of the internal resistance set: $0<$ Pint, I $\leq$ Pint, $n$. The profile of the Pint set is changeable by changing the value of the open circuit voltage, $U_{o} / V$. The trend line of the 12 input time values (Line 3 ), and the trend line of generated time set $\left(t_{1} \cdots t_{320}\right)$ (Line 4), are shown in Figure 3.

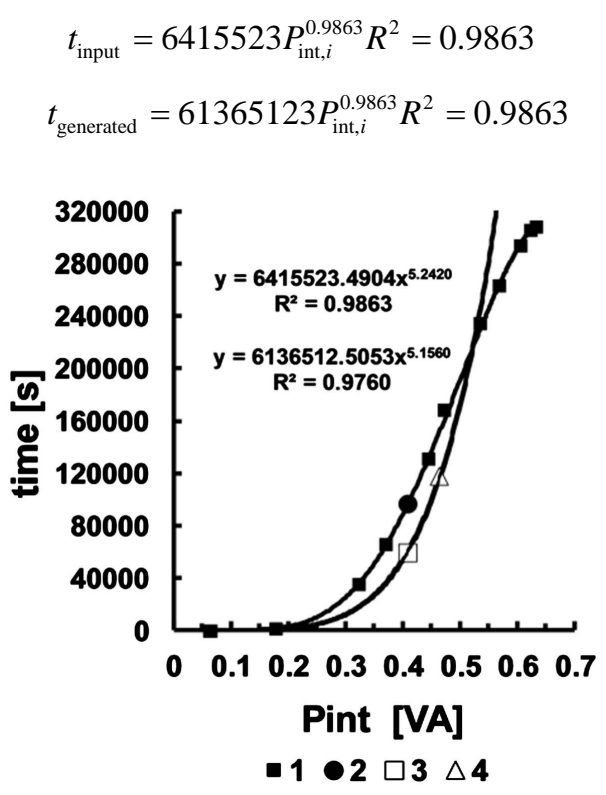

Figure 3. Input time and generated time vs. power of internal resistance, Pint/VA, LR20VARTA, $10 \mathrm{Ohm}, 1$. t/s-input data, 12 points-symbols; 2. t/s-generated discharge time, 320 points-line; 3. Trend line for input data: $t$ input, $I=415523$ (Pint, $i)^{\wedge 5.242 ;}$. Trend line for generated discharge time: $t$ generated, $i=6136512(\text { Pint, } i)^{\wedge 5.156 . ~}$ 
These values are very close to one another. By changing the coefficient and exponent, Equation (19a), the generated time profile changes but may not overlapped the 12 discharge input data profile (as well as the previously generated) time values, i.e., the CDCA basic equation is not obtained. In the next step, on the basis of the empirical equation ([1], Equation (17)), CDCA introduces the ratio of the adjacent points of (a) the initial power of internal resistance, Pint/VA (Line 1), (b) the trend line of input time, t/s (Line 2), and (c) the generated time values, t/s (Line 3 ) and puts these in relation vs. the ratio of Pint, $i /$ Pint, $i-1$ in logarithm form (Figure 4):

$$
\begin{gathered}
\text { Power of Internal Resistance }: \ln \left[\frac{P_{\mathrm{int}, i}}{P_{\mathrm{int}, i-1}}\right]=\ln \left[\frac{P_{\mathrm{int}, i}}{P_{\mathrm{int}, i-1}}\right] R^{2}=1 \\
\text { Input time }: \ln \left[\frac{t_{i}}{t_{i-1}}\right]=5.15 \ln \left[\frac{P_{\mathrm{int}, i i}}{P_{\mathrm{int}, i-1 i-1}}\right]-10^{-16} R^{2}=1 \\
\text { Generated time }: \ln \left[\frac{t_{i}}{t_{i-1}}\right]=7.0289 \ln \left[\frac{P_{\mathrm{in}, i i}}{P_{\mathrm{int}, i-1 i-1}}\right]-0.0141 R^{2}=.9988
\end{gathered}
$$

Thus, a linear relation between the input and generated time ratios is obtained. However, the " $S$ " profile of the 12 discharge input time values is not achieved by changing the coefficients in Equation (20b). Because of this fact, CDCA accepts, as a useful solution, the ratios of the adjacent ratios (cross-ratio) of both power and time, with the background that the adjustable power operator set generates a time operator set, i.e., time profiles that satisfy the input data may be achieved. The operator members, power and time, comprise the three successive values [1] [2] found at the " $i-2$ ”, “ $i-1$ ” and " $i$ ” positions. Both operators are found at the " $i$ " position. As the first step in time generation, CDCA defines the initial time operator comprising the following: (a) Experimental time from the solo common pair (at " $i$ "); (b) The time it takes to set up (at " $i-1$ "); and (c) The time value that is calculated (at “ $i-2$ ”). The next time operator member contains two known time values from the previous (forward or backward direction) operator member, and the third time value is calculated using the known power operator member. This algebraic operation, starting from the real solo common pair is performed to the end as well as from the very beginning of discharge. The discharge curve generated by the initial power operator set is based on the input data. Adjustment proceeds by changing the coefficients of the power operator set (Equation (22) until the next criterion is satisfied:

$$
t_{\text {input }, i}=b t_{\text {generated }, i}, \quad 0.99<b<1.01 \text { and } \quad 0.999<R^{2} \leq 1
$$

Based on this, the generated time set may be accepted as the CDCA discharge curve time vs voltage instead of the experimental voltage vs time. In accordance with such a result, CDCA accepts the four next forms of the CDCA basic equation:

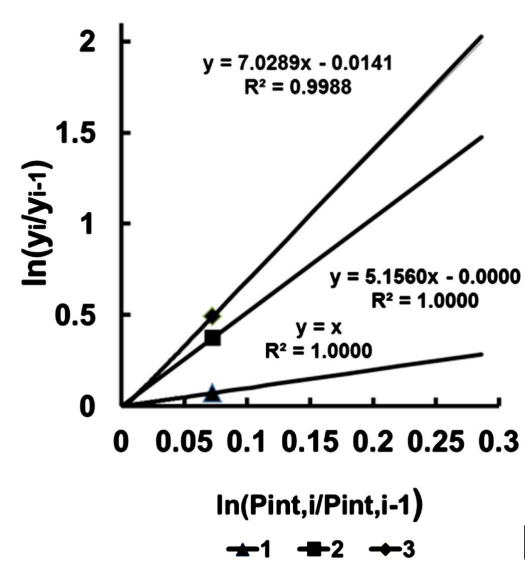

Figure 4. Linear dependence the ratios of the adjacent members, i.e. 1. The power of internal resistance, $\ln ($ Pint, $i /$ Pint,$i-1)=\ln ($ Pint, $i /$ Pint, $i-1) ; 2$. The trend line of the input data, in Figure $3, \ln (\mathrm{ti} / \mathrm{ti}-1)=$ $5.1560[\ln ($ Pint, $i /$ Pint, $i-1)]$; 3. Trend line for generated discharge time: $\ln (\mathrm{ti} / \mathrm{ti}-1)=7.0289[\ln ($ Pint, $i /$ Pint, $i-1)]-0.0141$ on the ratios of the power of the internal resistance adjacent members. 


$$
\begin{array}{ll}
\frac{\ln \frac{t_{i}}{t_{i-1}}}{\ln \frac{t_{i-1}}{t_{i-2}}}=\frac{\ln \left[\frac{P_{\mathrm{int}, i}}{P_{\mathrm{int}, i-1}}\right]}{\ln \left[\frac{P_{\mathrm{int}, i-1}}{P_{\mathrm{int}, i-2}}\right]} \quad(a) & \frac{\ln \frac{t_{i}}{t_{i-1}}}{\ln \frac{t_{i-1}}{t_{i-2}}}=\frac{a+b i+c i^{2}+d i^{3}}{A+B i+C i^{2}+D i^{3}} \\
\left.\frac{\Delta t_{i}}{\frac{t_{i-1}}{\Delta t_{i-1}}}=\frac{\left[\frac{\Delta P_{\mathrm{int}, i}}{t_{i-2}}\right.}{\left[\frac{P_{\mathrm{int}, i-1}}{\left[\frac{\Delta P_{\mathrm{in}, i-1}}{P_{\mathrm{int}, i-2}}\right]} \quad\right. \text { (c) }}\right] & \frac{\frac{\Delta t_{i}}{t_{i-1}}}{\frac{\Delta t_{i-1}}{t_{i-2}}}=\frac{a+b i+c i^{2}+d i^{3}}{A+B i+C i^{2}+D i^{3}}
\end{array}
$$

In addition to the power operator, CDCA introduces the polynomial ratio, Equations (22b), (22d). CDCA also defines generalized equations to interrelate (difference, sum, ratio, etc.) the CDCA battery characteristics.

$$
\frac{\frac{\Delta y_{i}}{y_{i-1}}}{\frac{\Delta y_{i-1}}{y_{i-2}}=\frac{\left[\frac{\Delta P_{\mathrm{int}, i}}{P_{\mathrm{int}, i-1}}\right]}{\left[\frac{\Delta P_{\mathrm{int}, i-1}}{P_{\mathrm{int}, i-2}}\right]} \quad \text { (a) }} \frac{\frac{y_{i}}{\sum_{1}^{1 i-1} y_{i-1}}}{\frac{y_{i-1}}{\sum_{1}^{1 i-2} y_{i-2}}}=\frac{\left[\frac{P_{\mathrm{int}, i}}{\sum_{1}^{i-1} P_{\mathrm{int}, i-1}}\right]}{\left[\frac{P_{\mathrm{int}, i}}{\sum_{1}^{1 i-2} P_{\mathrm{int}, i-2}}\right]}
$$

$$
\frac{\ln \frac{y_{i}}{y_{i-1}}}{\ln \frac{y_{i-1}}{y_{i-2}}}=\frac{\ln \left[\frac{P_{\mathrm{int}, i}}{P_{\mathrm{int}, i-1}}\right]}{\ln \left[\frac{P_{\mathrm{int}, i-1}}{P_{\mathrm{int}, i-2}}\right]}
$$

where " $y$ ” may be time and time-dependent parameters. Using Equation (22a), the next time calculation equations are obtained:

$$
\begin{aligned}
& t_{i}=t_{i-1}\left[\frac{t_{i-1}}{t_{i-2}}\right]\left[\frac{\ln \frac{P_{\mathrm{int}, i}}{P_{\mathrm{int}, i-1}}}{\ln \frac{P_{\mathrm{nn}, i-1}}{P_{\mathrm{int}, i-2}}}\right] \\
& t_{i-2}=t_{i-1}\left[\frac{t_{i-1}}{t_{i}}\right]\left[\frac{\ln \frac{P_{\mathrm{int}, i-1}}{P_{\mathrm{in}, i-2}}}{\ln \frac{P_{\mathrm{nn}, i}}{P_{\mathrm{int}, i-1}}}\right]
\end{aligned}
$$

Starting from the initial experimentally known time, the time set may be generated in the forward and backward directions. In the forward direction, the known values are " $i-2$ " and " $i-1$ ", whereas the third, " $i$ ”, is calculated. In the backward direction, the known values are " $i$ " and " $i-1$ ", whereas the third, " $i-2$ ", would be calculated. The backward generation leads to the very beginning of discharge. If a backward generation is performed from the final time, it is the verification of the forward generated curve. The precise adjusting of the time operator set (left-hand sides of Equation (22)) by changing the power operator set (right-hand sides of Equation (22)) leads to satisfying the linearity of input data to the generated discharge curve (Equation (21)). CDCM battery testing is extended and improved regarding the common parameters for electrochemical systems, discharge modes, and regimes.

\subsection{Operators in This Article}

In this article, using the input data, CDCA generates 20 operators (Figure 5), 20 discharge time curves (Figure 6), and, in addition, 20 sets of time sub-intervals (Figure 7). These results cover the following:

a Mono-cell battery LR20VARTA, 10 Ohm [1], 5 operator pairs: Equations 22(a)-(d), curves: 1 - 4 forward and Equation (22a), 2-backward direction;

b Multi-cell MALLORY batteries, 4 battery designs [2], 10 discharges, average cell, curves: 6 - 15 (Equation (22a));

c Multi-cell lead-acid battery, 6 cells, 5 constant current loads, average cell (Equation (22a)), curves: 16 - 20, and Figure 2.

The discharge curve can be shown either as a set of points, i.e., curve, or as a set of columns placed on the power plane and along the voltage, current and power lines. 


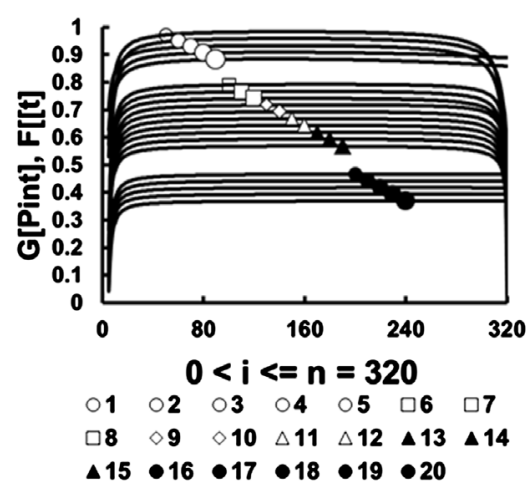

Figure 5. CDCA, time and power of internal resistance operators vs. n-set, multiplied by $1 \geq$ 0.375, LR20VARTA, 10 Ohm, Equation (22), line: 1. forward (a); 2. backward (a); 3. forward (b); 4. forward (c); 5. forward (d). MALLORY: constant resistive loads, Equation (22a), 9K62: 6. 50 Ohm; 7. 100 Ohm; 8. 250 Ohm, PX24: 9. 100 Ohm; 10. 160 Ohm, PX21: 11. 150 Ohm; 12. $250 \mathrm{Ohm}, 7 \mathrm{~K} 67: 13.100 \mathrm{Ohm}$; 14. $200 \mathrm{Ohm}$; 15. $500 \mathrm{Ohm}$. SONNENSCHEIN lead-acid battery, constant currents, Equation (22a): 16. 1.2 A, $[F(t)=G(P)] x 0.475$, 17. 2.4 A, $[F(t)=G(P)] x 0.45$, 18. 4.8 A, $F(t)=G(P)] x 0.425,19.7 .2 A[F(t)=G(P)] x 0.4,20.12,0 A,[F(t)=G(P)] \times 0.375$

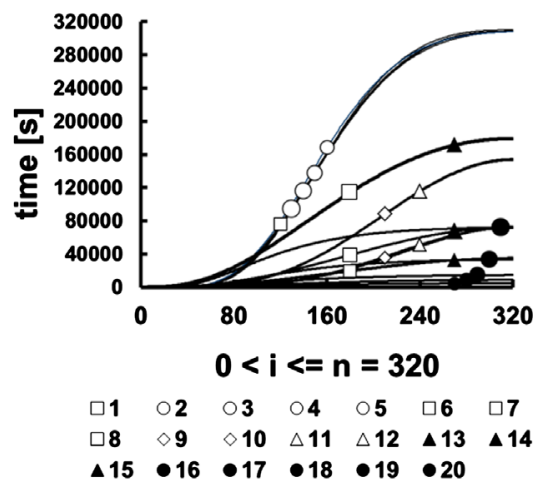

Figure 6. CDCA, discharge times vs. n-set, generated with operators in Figure 5, (Ohm/seconds): LR20VARTA, 10 Ohm, Equation (22), line:1-forward (a)/308,000s, 2-backward (a)/308,000s, 3-forward (b)/ 308,000s, 4-forward (c)/308,000s, 5-forward (d)/308,000s. MALLORY: constant resistive loads, Equation (22a), 9K62: 6.50 Ohm/35,200s; 7. 100 Ohm/71,200s; 8. 250 Ohm/179,200s. PX24: 9. 100 Ohm/ 72,000s, 10. $160 \mathrm{Ohm} / 154,280 \mathrm{~s}$. PX21: 11. $150 \mathrm{Ohm} / 72,000 \mathrm{~s} ; 12$. $250 \mathrm{Ohm} / 154,280 \mathrm{~s}$. 7K67: 13. $100 \mathrm{Ohm} / 35,200 \mathrm{~s}$, 14. $200 \mathrm{Ohm} / 71,200 \mathrm{~s} ; 15.500 \mathrm{Ohm} / 179,200 \mathrm{~s}$. SONNENSCHEIN, constant currents, Equation (22a): 16. $1.2 \mathrm{~A} / 72,000 \mathrm{~s} ; 17.2 .4 \mathrm{~A} / 33,320 \mathrm{~s} ; 18.4 .8 \mathrm{~A} / 14,400 \mathrm{~s} ; 19.7 .2 \mathrm{~A} / 8780 \mathrm{~s} ; 20.12 .0 \mathrm{~A} / 4600 \mathrm{~s}$.

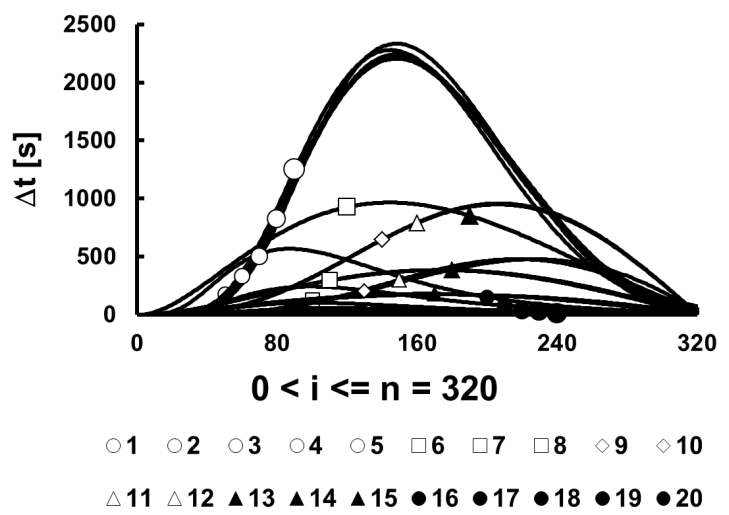

Figure 7. Discharge time sub-intervals vs. n-set, LR20VARTA 10 Ohm, curves: 1, 2, 3, 4, 5, MALLORY: 9K62, curve/Ohm: 6/50, 7/100, 8/250, PX24, curve/Ohm: 9/100, 10/160, PX21, curve/Ohm: 11/150, 12/250, 7K67, curve/ Ohm: 13/100, 14/200, 15/500, SONNESCHEIN, curve/current: 16/1.2A, 17/2.4A, 18/4.8A, 19/7.2A, 20/12A. 


\section{CDCA Battery Characteristic}

Currently, many electrochemical systems exist, and most can be used reliably in a wide range of applications. If the average cell represents a battery of one of the electrochemical systems, the closed discharge voltage interval of the cell is the valid abscissa for any battery design. In addition to voltage, $U_{o}>V_{i} \geq V_{\text {final }}$, and the overvoltage set, $0<\eta_{i} \leq \eta_{\text {final }}$, CDCM introduces the $N$-n-set, $n=U_{o} /(2 \Delta V)=N / 2$, as the common abscissa for every electrochemical system. The discharge time and battery characteristics depend on the battery state of charge, state of health, and discharge conditions. The CDCA algebraic equations of battery characteristics, the (a) voltage and current capacities and (b) energy volumes, are based on the voltage interval and battery time response. The time response includes the discharge time and time sub-intervals. Numerical summations are performed across the interval $0<i \leq n$.

\subsection{Time Voltage and Current Surfaces-Capacities}

The overall voltage capacity, $U_{o} t / V s$, is divided into two parts: voltage on load, $S_{v, i} / V_{s}$, and overvoltage, $S_{\eta, i} / V_{s}$, as voltage losses:

$$
\begin{gathered}
U_{0} t_{i}=V_{i} t_{i}+\eta_{i} t_{i}=\sum_{1}^{i} V_{i} \Delta t_{i}+\sum_{1}^{i} \Delta V_{i} t_{i}+\sum_{1}^{i} \eta_{i} \Delta t_{i}+\sum_{1}^{i} \Delta \eta_{i} t_{i}=\sum_{1}^{i} V_{i} \Delta t_{i}+\sum_{1}^{i} \eta_{i} \Delta t_{i} \\
S_{V, I}=\sum_{1}^{i} V_{i} \Delta t_{i}=V_{i} t_{i}+\sum_{1}^{i}|\Delta V| t_{i}=U_{0} t_{i}-\sum_{1}^{i} \eta_{i} \Delta t_{i} \\
S_{\eta, I}=\sum_{1}^{i} \eta_{i} \Delta t_{i}=\eta_{i} t_{i}+\sum_{1}^{i}|\Delta \eta| t_{i}=U_{0} t_{i}-\sum_{1}^{i} V_{i} \Delta t_{i}
\end{gathered}
$$

The overall current capacity and parts are obtained by multiplying Equations (26)-(28) with the reciprocal of the constant resistive load, $\mathrm{Rl} / \mathrm{Ohm}$ :

$$
\begin{gathered}
I_{0} t_{i}=I_{i} t_{i}+v_{i} t_{i}=\sum_{1}^{i} I_{i} \Delta t_{i}+\sum_{1}^{i} \Delta I_{i} t_{i}+\sum_{1}^{i} v_{i} \Delta t_{i}+\sum_{1}^{i} \Delta v_{i} t_{i}=\sum_{1}^{i} I_{i} \Delta t_{i}+\sum_{1}^{i} v_{i} \Delta t_{i} \\
Q_{I, i}=\sum_{1}^{i} I_{i} \Delta t_{i}=I_{i} t_{i}+\sum_{1}^{i}|\Delta I| t_{i}=I_{0} t_{i}-\sum_{1}^{i} v_{i} \Delta t_{i} \\
Q_{I, i}=\sum_{1}^{i} v_{i} \Delta t_{i}=v_{i} t_{i}+\sum_{1}^{i}|\Delta v| t_{i}=I_{0} t_{i}-\sum_{1}^{i} I_{i} \Delta t_{i}
\end{gathered}
$$

\subsection{OEV Energy Volumes}

CDCA places the discharge times, $t_{i} / s$, as the parallelepiped's altitudes on the power plane to define the energy's parts in the form of parallelepipeds:

$$
U_{0} I_{0} t_{i}=\left(V_{i}+\eta_{i}\right)\left(I_{i}+v_{i}\right)=P_{\text {load }, i} t_{i}+P_{\text {ext }, i} t_{i}+P_{\text {int }, i} t_{i}+P_{\mathrm{CDCM}, i} t_{i}
$$

Overall on load current voltage CDCM losses.

To define the OEV parts, CDCA places discharge time, $0<t_{i} / s<t_{n} / s$ i.e., the sum of time sub-intervals, $0<\Sigma \Delta t_{i} / s<\Sigma \Delta t_{n} / s$, along power lines: on load, external, internal and CDCM losses in Equation (32) and calculates volumes by summation by parts:

$$
\begin{aligned}
U_{0} I_{0} t_{i}= & \sum_{1}^{i}\left|\Delta P_{\text {load }, i}\right| t_{i}+\sum_{1}^{i}\left|P_{\text {load }, i} \Delta t_{i}\right|+\sum_{1}^{i}|| \Delta P_{\text {ext }, i}|| t_{i}+\sum_{1}^{i} P_{\text {ext }, i} \Delta t_{i}+\sum_{1}^{i}\left|\Delta P_{\text {int }, i}\right| t_{i}+\sum_{1}^{i} P_{\text {int }, i} \Delta t_{i} \\
& +\sum_{1}^{i}\left|\Delta P_{\mathrm{CDCM}}\right| t_{i}+\sum_{1}^{i} P_{\mathrm{CDCM}, i} \Delta t_{i} .
\end{aligned}
$$

where

$$
\sum_{1}^{i}\left|\Delta P_{\text {load }, i}\right| t_{i}=\sum_{1}^{i}\left|\Delta P_{\mathrm{int}, i}\right| t_{i}+\sum_{1}^{i}\left\|\Delta P_{\text {ext }, i}\right\| t_{i}++\sum_{1}^{i} P_{\mathrm{CDCM}, i} \Delta t_{i}
$$




$$
U_{0} I_{0} \sum_{1}^{i} \Delta t_{i}=\sum_{1}^{i} V_{i} I_{i} \Delta t_{i}+\sum_{1}^{i} \eta_{i} I_{i} \Delta t_{i}+\sum_{1}^{i} V_{i} v_{i} \Delta t_{i}+\sum_{1}^{i} \eta_{i} v_{i} \Delta t_{i}
$$

Using Equation (35), the OEV parallelepiped is defined as the sum of the " $n$ " horizontal layers that contain the four electrochemical parts, and each of the parts has the same time sub-interval:

$$
\begin{gathered}
E_{\text {load }, i}=\sum_{1}^{i} \Delta E_{\text {load }, i}=\sum_{1}^{i} V_{i} I_{i} \Delta t_{i}+\sum_{1}^{i} \Delta S_{V, i} I_{i}+\sum_{1}^{i} V_{i} \Delta Q_{I, i} \\
E_{\mathrm{int}, i}=\sum_{1}^{i} \Delta E_{\mathrm{int}, i}=\sum_{1}^{i} \eta_{i} I_{i} \Delta t_{i}=\sum_{1}^{i} V_{i} \Delta Q_{i} \\
E_{\mathrm{ext}, i}=\sum_{1}^{i} \Delta E_{\mathrm{ext}, i}=\sum_{1}^{i} V_{i} v_{i} \Delta t_{i}+\sum_{1}^{i} \Delta S_{V, i} v_{i} \\
E_{\mathrm{CDCM}, i}=\sum_{1}^{i} \Delta E_{\mathrm{CDCM}, i}=\sum_{1}^{i} \Delta \eta \Delta v \Delta t_{i}
\end{gathered}
$$

The applied electrochemical model and time sub-intervals configure the horizontal layers.

\subsection{Discharge Time and Time Sub-Intervals vs $n$ - $N$-Set}

Treating the discharge time, Equation (10), and time sub-intervals, Equation (11), as the range vs. $N$ - $n$-set as the domain, CDCA defines the following relations:

$$
\begin{array}{llr}
i \Delta t_{i}=t_{i}+\sum_{1}^{i} i \Delta\left(\Delta t_{i}\right) \quad(a) \quad & i t_{i}=\sum_{1}^{i} t_{i}+\sum_{1}^{i} i \Delta t_{i} \quad(b) \\
i^{2} \Delta t_{i}=i t_{i}+i \sum_{1}^{i} i \Delta\left(i \Delta t_{i}\right) \quad(c) & i^{2} t_{i}=i \sum_{1}^{i} t_{i}+i \sum_{1}^{i} i \Delta t_{i}
\end{array}
$$

The electrochemical meanings of these equations are obtained by multiplying Equation (40) by constant voltage/current steps $(\Delta V, \Delta \eta, \Delta I, \Delta v)$, as well as by summation, to define capacity surfaces and energy volumes. Further analyses of these relations are not addressed in this article. The discharge time and the time sub-interval curves are shown in Figure 6 and Figure 7.

\subsection{Battery Average Characteristics}

CDCA introduces the battery average characteristics [1] [2] that transform the irregular surfaces and volumes of the real discharge into the regular quadrilateral form of the virtual discharges, with their extents unchanged. By multiplying Equation (26) with $\left(t_{i} / t_{i}\right)=1$, the overall voltage capacity is divided into the average voltage and overvoltage parallelograms of the virtual discharge:

$$
\sum_{1}^{i} U_{0} \Delta t_{i}=\frac{t_{i}}{t_{i}}+\left|\sum_{1}^{i} V_{i} \Delta t_{i}+\sum_{1}^{i} \eta_{i} \Delta t_{i}\right|=V_{\text {avg }, i} t_{i}+\eta_{\text {avg }, i} t_{i}
$$

At any state of discharge, the average voltage, $V_{\text {avg }, i} / V$, is the straight line of the changing discharge curve of " $S$ " shape. This means that the virtual discharge with the constant average voltage produces the same voltage capacity as the real discharge by the discharge curve of " $S$ " shape. The average voltages of discharge in this article are shown in Figure 8. Multiplying Equation (41) with the reciprocal of load resistance, $(\mathrm{Rl} / \mathrm{Ohm})^{-1}$, as well as multiplying Equation (29) with $\left(t_{i} / t_{i}\right)=1$, the average discharge current and current losses are obtained:

$$
\sum_{1}^{i} I_{0} \Delta t_{i}=\frac{t_{i}}{t_{i}}\left|I_{i} \Delta t_{i}+\sum_{1}^{i} v_{i} \Delta t_{i}\right|=I_{\mathrm{avg}, i} t_{i}+v_{\mathrm{avg}, i} t_{i}
$$

The actual power on load, VI/VA and battery average power, $v_{\text {avg }, i} I_{\text {avg }, i}$, are shown in Figure 1 . The known average voltage and current define the average overvoltage and average current losses:

$$
\eta_{\text {avg }, i}=U_{0}-V_{\text {avg }, i}(a) \quad v_{\text {avg }, i}=I_{0}-I_{\text {avg }, i}
$$




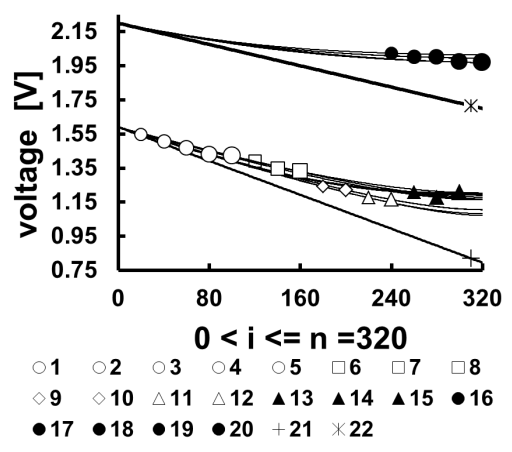

Figure 8. Average voltages, $V_{\text {avg, } i} / V$ : LR20VARTA 10 Ohm, curves: 1, 2, 3, 4, 5, MALLORY:9K62, curve/

Ohm: 6/50, 7/100, 8/250, PX24, curve/Ohm: 9/100, 10/160, PX21, curve/Ohm: 11/150, 12/250, 7K67, curve/ Ohm: 13/100, 14/200, 15/500, Sonneschein: curve/current: 16/1.2A, 17/2.4A, 18/4.8A, 19/7.2, 20/12A Average cell voltages, Vcell/V: line 21 primary batteries, line 22 lead acid battery.

In parallel with the electrochemical parameters, the parameters in Equations (42)-(43) divide the battery power plane (Figure 1) into the four analogue average power values: on load, external, internal and CDCM, i.e.

$$
\begin{array}{lll}
P_{\text {avg,load }, i}=V_{\text {avg }, i} I_{\text {avg }, i} & (a) & P_{\text {avg, }, \text { nt }, i}=\eta_{\text {avg }, i} I_{\text {avg }, i}
\end{array}
$$

Multiplying Equation (35) with $\left(t_{i} / t_{i}\right)=1$, the next equations are obtained:

$$
\begin{gathered}
U_{0} I_{0} \sum_{1}^{i} \Delta t_{i}=\frac{t_{i}}{t_{i}}\left|\sum_{1}^{i} P_{\text {load }, i} \Delta t_{i}+\sum_{1}^{i} P_{\text {ext }, i} \Delta t_{i}+\sum_{1}^{i} P_{\text {int }, i} \Delta t_{i}+\sum_{1}^{i} P_{\mathrm{CDCM}, i} \Delta t_{i}\right| \\
U_{0} I_{0} \sum_{1}^{i} \Delta t_{i}=t_{i} P_{\text {avg, load }, i}+t_{i} P_{\text {avg,ext }, i}+t_{i} P_{\text {avg, }, \text { nt }, i}+P_{\text {avg, CDCM }, i} t_{i}
\end{gathered}
$$

A battery OEV is represented by the three figures: (a) the four electrochemical right parallelepipeds, Equation (32); (b) the sum of $1<i \leq n$ horizontal time sub-interval layers, Equation (35); and (c) the sum of the four CDCA averaged parallelepipeds, Equation (44). All of these parallelepipeds change simultaneously and successively due to the power values and the time sub-intervals changing. Combining these three figure using algebraic solutions, CDCA enables recalculation of the real discharge into virtual discharges in accordance with Table 1 in this article.

\subsection{CDCA 3D Curve, Surface and Space}

In addition, CDCA can represent the battery characteristics in a 3D chart where the capacity surfaces and energy volumes are visible. In this article, the LR20VARTA, Mallory primary batteries, and lead acid battery are shown in Figures 9-11, respectively. In the created 3D charts, every tenth altitude of $n=320$ is drawn.

The LR20VARTA discharge is presented by the five time sets. The first two are plotted at the IPP at $0.00 \mathrm{~A}$ and 0.08 A constant current lines. The two next sets are plotted at the 0.0 and $0.8 \mathrm{~V}$ constant voltage lines. The sets between both pairs, current and voltage, are not plotted due to visibility of the chart, but they are discharge surfaces in an "S" shape. The fifth set is plotted at the power-time (overvoltage-current losses, i.e., voltage-current) line. The final time altitudes, tn/s, cover one-quarter of the IPP, $U_{o} I_{o} / 4$. The energy on load volume is in the form of a truncated pyramid with the two curved surfaces in the " $S$ " shape. The differences between the OEV and energy on load are energy losses (which consist of the three parts) and are positioned in the "empty" upper half of the parallelepiped. These real discharge volumes may be recalculated for other discharge modes and regimes.

3D OEVs for the 10 MALLORY discharges are placed at the final average currents as voltage-time surfaces (Figure 10). Discharge altitudes are started at $U_{o} / V$ and are positioned step by step, changing overvoltage and time, toward the $0.8 \mathrm{~V}$ and final time. Finally, OEVs are formed as prisms with bases that are time-overvoltage surfaces, and the third dimension is the constant final average current value. 


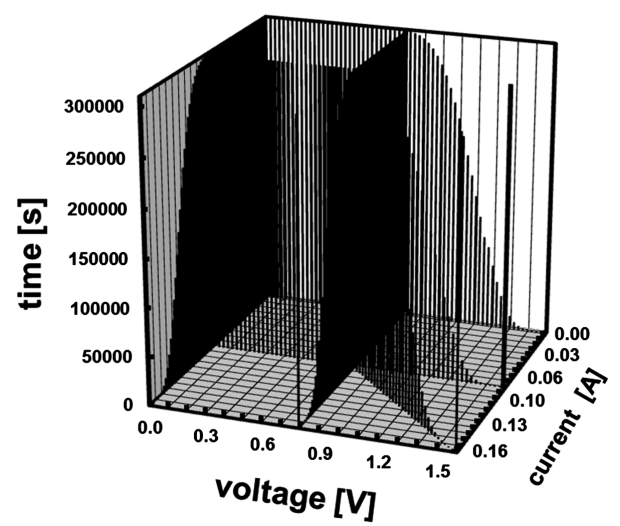

Figure 9. 3D figures of discharge time sets (every tenth altitude), Equation (22a), LR20VARTA, 10 Ohm, $t_{\text {final }}=308000 \mathrm{~s}$, repeated along: (a) constant voltage: $0.8 \mathrm{~V}$ and $0 \mathrm{~V}$, (b) constant current: $0.08 \mathrm{~A}$ and $0 \mathrm{~A}$; (c) discharge power: $U_{o} I_{o}>V I \geq U_{o} I_{o} / 4$.

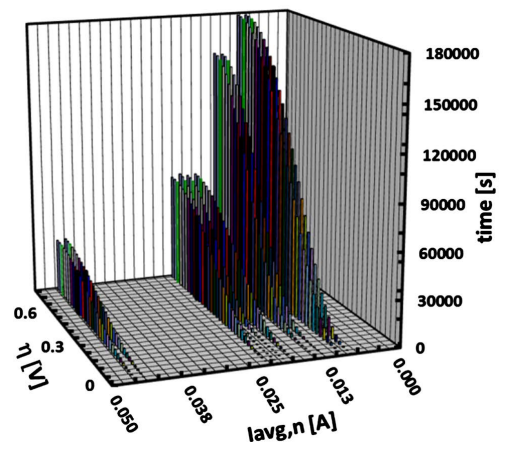

Figure 10. 3D figures of discharge time sets (every tenth altitude) placed at final average currents, Iavg, n/A:, Equation (22a), MALLORY: 0.046094/34,714s, 0.023438/71,201s, 0.010156/179,200s. PX24: 0.021875/ 72,000s, 0.014844/154,280s. PX21: 0.023050/72,000s, 0.019281/154,000s, 7K67: 0.044531/35,194s. 0.018750/ 71,200 s, $0.008599 / 179,200$ s.

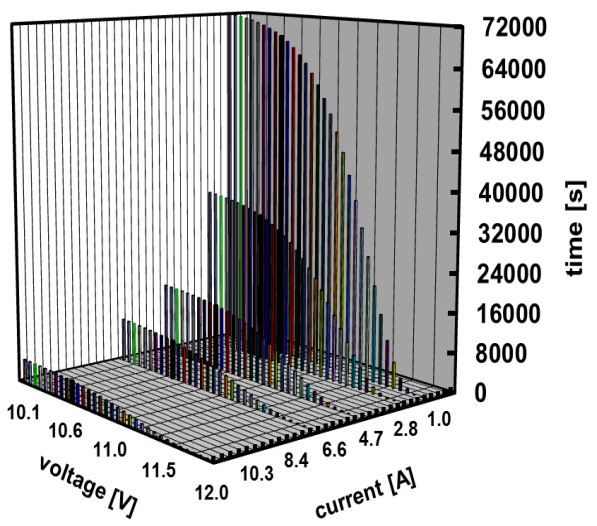

Figure 11. 3D figures of discharge timeset, t/s (every tenth altitude), Equation (22a), lead acid VRLAS onnenschein Dryfit A 51/24.0, placed at constant discharge currents: I/A: 1.2, 2.4, 4.8, 7.2 and 12.0.

3D OEVs for five lead-acid VRLA discharges (Figure 11) are placed as with the MALLORY discharges, i.e., the voltage capacity surfaces are placed at the real constant discharge currents, i.e., the third dimensions of the formed prisms.

For the 3D battery model, the necessary set of equations is described. The four following equations are recommended for creative and more detailed analysis of an actual OEV: 


$$
\begin{array}{llll}
E_{V I, i} / Q_{I, I}=S_{v, i} / t_{i} & \text { (a) } & E_{\eta, i} / Q_{I, i}=S_{\eta, i} / t_{i} & \text { (b) } \\
E_{V v, i} / Q_{v, i}=S_{v, i} / t_{i} & \text { (d) } & E_{\eta v, i} / Q_{v, i}=S_{\eta, i} / t_{i} & \text { (d) }
\end{array}
$$

The presented CDCM approach to battery testing and 3D battery models may be further improved with regard to the electrochemical model, operator meanings, and possible battery discharge prediction. The mathematical calculations were performed using Microsoft Excel software.

\section{Conclusions}

The calculated discharge curve method (CDCM) accepts that a mono- and multi-cell battery (C/B) is a physicochemical, thermodynamic, and electrochemical system in which the energy delivered is the sum of the useful work and energy losses. C/B testing is conducted in defined conditions, and the process is recorded as voltage changes over time. CDCM defines a battery average cell to represent a multi-cell battery and compares the average with the mono-cell or single cell separated from a serial and/or parallel connection. The battery operating conditions are classified using the load modes (resistor, current, voltage, power), regimes (self and device driving), and timing (continual and periodical).

The open-circuit voltage and initial current (defined by the resistive load) are the principal characteristics of a battery electrochemical system, and their intervals are divided into " $N$ " constant steps. Both the voltage and current sets define the battery initial power plane (IPP), which comprises $N^{2}$ smallest power parallelograms, which are the basis of the 3D battery model, i.e., the overall energy volume (OEV). The IPP comprises the power on load and powers of internal, external and CDCM resistances that appear at the discharge starts. During battery discharging, the power on load decreases, whereas the loss power increases, i.e., $N^{2}$ parallelograms are distributed, step by step, between the four parts of the IPP.

The battery discharge is recorded in the form "voltage vs. time", where time is the independent variable. CDCM was evolved from the Calculated Discharge Curve Algorithm (CDCA) as a mathematical tool. CDCA defines the basic equation by which discharge data from "voltage vs. time" transforms into "time vs. voltage". The result is the ordered triple cardinal number-voltage-time and is valid for all battery designs and electrochemical systems. This ordered triple defines a 3D mathematical model of OEV, i.e., the battery reversible energy volume. All parameters that define and are necessary to analyze OEV remain in correspondence with the initial ordered triple.

The basic equation is based on the three-point operator set that comprises the three successive values of the power of internal resistance, time, and time-dependent parameters. The operator set member is placed as the last of the three power and time values, and they are in correspondence, one to one, with all CDCA sets. The two earlier values of the operator exist in the two previous power and time operators. The basic equation generates the discharge time set, which overlaps the experimental time values and is accepted by CDCM for characterizing the battery during its exploitation. The discharge time is discharge duration and comprises the set of time subintervals, which are the duration of the voltage step change. In accordance with the " $S$ " shape of any discharge curve, the longest time sub-interval lies between the shortest initial and final time sub-interval. Using the timegeneration procedure, the 3D battery model is quantified, and CDCA can calculate the battery standard and new CDCM characteristics.

For averaging capacities, i.e., voltage/current-time surface to time and energy capacities, CDCA introduces average voltage, overvoltage, current and current losses. The battery average parameters transform the irregular forms of the OEV (capacities and volumes) into regular forms, i.e., parallelograms and parallelepipeds. All battery types of the same electrochemical system may be presented on the common voltage abscissa. The common abscissa for the batteries of all of the electrochemical systems is a cardinal number set.

The three-point operators, including time and time-dependent parameters, are the new battery characteristics, and they are not examined in this article. Examination of the interrelations of these battery operator interrelations may lead to new battery testing parameters.

\section{References}

[1] Djordjevic, A.B. and Karanovic, D.M. (1999) Cell Testing by Calculated Discharge Curve Method. Journal of Power Sources, 83, 134-140. http://dx.doi.org/10.1016/S0378-7753(99)00287-6

[2] Djordjevic, A.B. and Karanovic, D.M. (2006) Battery Testing by Calculated Discharge-Curve Method-Constant Re- 
sistive Load Algorithm. Journal of Power Sources, 162, 920-926. http://dx.doi.org/10.1016/j.jpowsour.2005.07.013

[3] Vincet, C.A. and Scrosati, B. (1997) Modern Batteries. Arnold, London.

[4] Bard, A.J. and Faulkner, R. (1980) Electrochemical Methods. John Wiley \& Sons, Inc., New York.

[5] Akay, T.J. (1980) Applied Numerical Methods for Engineers. John Wiley \& Sons, Inc., New York.

[6] Wu, M.-S., Lin, C.-Y., Wang, Y.-Y., Wan, C.-C. and Yang, C.R. (2006) Electrochimica Acta, 52, 1349-135.

\section{Nomenclature}

\section{List of symbols}

$E$ : energy (VAs)

Io: initial current (A)

I: current (A)

$\Delta I$ : constant current step (V)

$N$ : cardinal number set, $0<i<n<N$

$Q$ : current capacity (As)

$\mathrm{Rl}$ : constant resistance (Ohm)

$S$ : voltage capacity, Vs

$T$ : time (s)

$U_{o}$ : open circuit voltage (V)

$V$ : voltage (V)

$\Delta V$ : constant voltage step (V)

$x$ : common symbol

$y$ : common symbol

$\eta$ : overvoltage (V)

$v$ : current losses (A)

\section{Subscripts}

avg: averaging with time

ext: external energy losses

$i$ : discharge progress steps

$n$ : final step, $1<i \leq n<N$

int: internal losses

$o$ : refer to initial state 
Scientific Research Publishing (SCIRP) is one of the largest Open Access journal publishers. It is currently publishing more than 200 open access, online, peer-reviewed journals covering a wide range of academic disciplines. SCIRP serves the worldwide academic communities and contributes to the progress and application of science with its publication.

Other selected journals from SCIRP are listed as below. Submit your manuscript to us via either submit@scirp.org or Online Submission Portal.
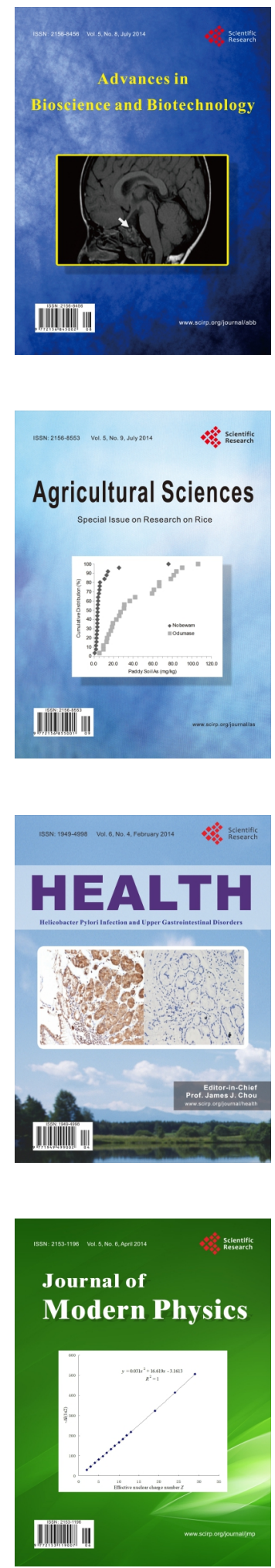
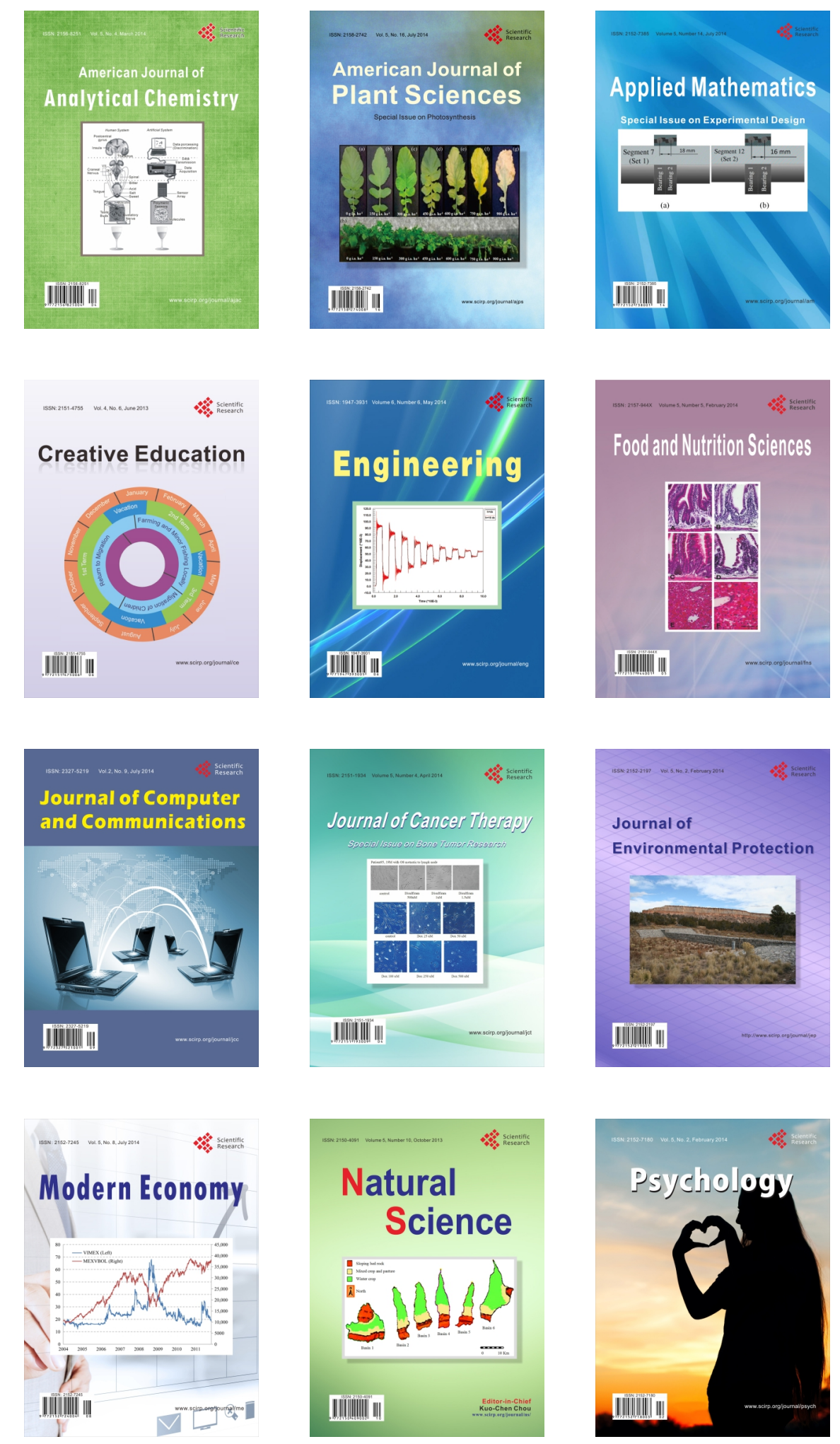